\title{
Modelo de enseñanza y aprendizaje significativo de los conceptos ácido-base con uso de NTIC
}

\section{Model of teaching and learning the concepts of significant acid-base with use of NTIC}

\section{Carlos Ariel Rentería Jiménez* \\ RESUMEN}

Esta comunicación recoge los resultados de investigación obtenidos con la aplicación de una estrategia pedagógica y didáctica de enseñanza de la química, fundamentada principalmente en el diseño, implementación y evaluación de una página web de conceptos ácido-base, usando situaciones problema con apoyo de recursos informáticos como instrumentos de aprendizaje, la cual está centrada principalmente en la enseñanza y el aprendizaje significativo de los conceptos quimicos relacionados con los ácidos y las bases.

Palabras clave: Aprendizaje significativo; Conceptos ácido-base; Ideas alternativas; Red conceptual; NTIC.

\begin{abstract}
This communication picks up the investigation results obtained with the application of a pedagogic strategy and didactics of teaching of the chemistry, based mainly in the design, implementation and evaluation of a page web of concepts acid-base, using situations problem with support of computer resources as learning instruments; which is centered mainly in the teaching and the significant learning of the chemical concepts related with the acids and the bases.
\end{abstract}

Keyword: Significant learning; Concepts acid-base; Alternative ideas; Conceptual net; NTIC.

\section{INTRODUCCIÓN}

En el campo de la investigación educativa se admite, desde hace varios años, la necesidad de utilizar los programas de ordenador de todo tipo en la enseñanza de la ciencias, por las indudables ventajas pedagógicas que se han ido poniendo de manifiesto en múltiples trabajos de divulgación e investigación realizados en los países más avanzados y, sobre todo, en el mundo anglosajón (Hartley 1988; Lelouche, 1998). En tales trabajos se ha puesto de manifiesto que los programas didácticos de ordenador poseen la propiedad de simular fenómenos naturales dificiles de observar en la realidad o de representar modelos de sistemas físicos inaccesibles facilitando su comprensión, ofreciendo además la posibilidad de llevar a cabo un proceso de aprendizaje y evaluación individualizada, entre otras muchas aplicaciones educativas.

Es conveniente desarrollar un método y estrategia docente que permita utilizar los recursos informáticos y las situaciones problemas como instrumentos de aprendizaje significativo de los conceptos químicos, en este caso, los conceptos ácido-base.

Se propone, el estudio del uso de situaciones problemas para el aprendizaje significativo de los conceptos ácido-base, bajo el contexto de las NTIC 


\section{Bioetnia Volumen 5 No 1 (enero-junio), 2008}

como modelo didáctico para la enseñanza de la química, desde una perspectiva multidisciplinar, que reúna las dimensiones ideas alternativas, resolución de situaciones problemas y asimilación conceptual. Esta perspectiva integral nos llevará en un primer momento a analizar la ideas alternativas que presentan los estudiantes acerca de los conceptos ácido-base, analizados desde la epistemología.

En segundo momento, se hace un análisis del proceso de resolución de problemas, la cual se identifica como una actividad crucial en las ciencias, además de ser inherente a la vida diaria y al trabajo profesional de los individuos (Garret, 1989), los cuales junto al desarrollo de las actitudes hacia las ciencias en los individuos, puede ser generado por procesos de intervención pedagógica que impliquen una enseñanza adecuada de las ciencias. En tercer lugar, se hace un recorrido histórico-crítico sobre los indudables beneficios que representa el uso de las nuevas tecnologías de la información y la comunicación en la didáctica de las ciencias. En cuarto lugar, se analizan los aspectos más generales acerca de la utilización de redes conceptuales, así como la utilización de Nuevas Tecnologías para la enseñanzaaprendizaje de la ciencia, en especial la química.

Por otra parte este estudio llevó a precisar aspectos relacionados con la metodología a aplicar en la investigación, que se fundamentó sobre las variables ideas alternativas, asimilación conceptual, resolución de situaciones problema, diseño y evaluación de una página web, la cual se desarrolló en cuatro (4) fases que denominadas fase inicial, fase de acomodación al cambio didáctico, fase de ejecución y fase de evaluación.

Se procedió luego a recoger la información, y se hizo el análisis de los resultados obtenidos con la aplicación de los distintos instrumentos utilizados para la recolección de la información, lo cual consistió en el análisis de las respuestas dadas por los alumnos de la muestra seleccionada a los dos cuestionarios propuestos, así como a la construcción de las redes conceptuales, antes y después de la aplicación de la estrategia didáctica propuesta.

Finalmente, se establecieron las conclusiones a las cuales nos llevó este trabajo de investigación, el aprendizaje significativo de los conceptos ácido-base, bajo el contexto de las NTIC.

Cabe mencionar que esta investigación recoge los resultados obtenidos con el proyecto duran- te el período 2005-2006. El desarrollo del proyecto se realizó con los estudiantes de primer semestre del programa de Biología, con Énfasis en Recursos Naturales, de la Universidad Tecnológica del Chocó "Diego Luis Córdoba", se trató de una propuesta en la que se hicieron objeto de trabajo en el aula los contenidos curriculares (conceptos ácido-base) recomendados para los estudiantes de programas de Biología, desde la perspectiva didáctica del aprendizaje significativo a partir de situaciones problema, bajo el apoyo de las NTIC.

El origen de este trabajo es la preocupación por los bajos resultados en la asignatura de química general del Programa de Biología, con Énfasis en Recursos Naturales, de la Universidad Tecnológica del Chocó. Es fundamental aplicar otros métodos de enseñanza-aprendizaje diferentes (como alternativa a los métodos tradicionales), que permitan una mejor asimilación de conceptos relacionados con la química, en especial con la química de los ácidos y de las bases.

\section{PLANTEAMIENTODEL PROBLEMA}

Uno de los objetivos fundamentales de la didáctica de las ciencias es buscar nuevas formas de enseñanza que promuevan una mayor participación por parte del estudiante en el proceso de enseñanza-aprendizaje; sin embargo, en Colombia y sobre todo en el departamento de Chocó, es muy común que la enseñanza esté completamente centrada en el docente, lo cual implica varias limitaciones, pero vamos a referirnos principalmente a los problemas surgidos cuando los estudiantes se enfrentan a situaciones problemas.

La mayoría de los estudiantes no utilizan representaciones válidas del problema ni de las posibles vías para su resolución antes de iniciar esta resolución. Es así como "la mayoría de los estudiantes no elaboran gráficos o diagramas para ayudarse en la comprensión del problema y la mayoría de ellos tampoco piensa acerca de las diferentes formas y métodos que pueden existir para resolver un problema (Fortunato, Álvarez, 2000). Entre otras, y es aquí donde las NTIC constituyen una herramienta de apoyo al conocimiento y aprendizaje de las ciencias naturales en especial a la química y se hace necesario buscar de manera progresiva su integración con el currículo de ciencias químicas lo cual además de crear una visión más amplia y completa, y de 
hacerla más eficiente, propicia el trabajo en equipo, la participación y los aprendizajes se centran significativamente alrededor de procesos integrados más amplios, que demandan la comprensión de los estudiantes y el desarrollo de diferentes habilidades a través del uso y aplicación de situaciones problemas bajo el contexto de las NTIC como un modelo didáctico para la enseñanza de la química.

Las dificultades de aprendizaje frente a la resolución de problemas en química y su correlato de fracasos escolares reconocen como factores fundamentales las insuficiencias en los procesos de comunicación docente y la pobre decodificación de su discurso por los alumnos, limitaciones propias de los recursos y materiales didácticos empleados (por ejemplo, presentación de contenidos disciplinares descontextualizados), así como el tipo y calidad de las evaluaciones utilizadas para detectar los avances en los procesos de aprendizaje (predominio de evaluaciones finales, cuantitativas y sumativas frente a evaluaciones formativas y de proceso). Desde una concepción de aprendizaje investigativo, podemos añadir que los resultados finales de la evaluación de los aprendizajes escolares estarán indudablemente sesgados por las características socio-ambientales específicas del grupo de clase, condicionantes a menudo de actitudes negativas de rechazo hacia las ciencias, y por las fuertes divergencias entre el lenguaje de la ciencia profesional, el de la ciencia escolar y el lenguaje cotidiano de los alumnos (Izquierdo et al., 2000).

Considerando las ideas planteadas, el problema se enuncia a través de las siguientes preguntas de investigación:

¿Qué ideas alternativas manejan los estudiantes objeto de esta investigación en torno a los conceptos ácido-base?

¿Es posible que el diseño, la implementación y la evaluación de una página web que incluya actividades interactivas, con situaciones problema sobre los conceptos ácido-base, contribuya de manera notoria a un aprendizaje más significativo en torno a los conceptos ácido-base?

El objetivo fundamental de este estudio fue el de implementar un modelo de enseñanza-aprendizaje de la química con el uso de situaciones problemas, apoyado en un sitio web con recursos, actividades y estrategias para docentes y estudiantes de primeros semestres universitarios en el área de química general, ofrecida por algunos programas de la Universidad Tecnológica del Chocó "Diego Luis Córdoba en Quibdó, Colombia, además, poder identificar algunas concepciones que poseen los estudiantes de primer semestre de Biología, con Énfasis en Recursos Naturales.

\section{ANTECEDENTES}

Son pocos los trabajos que se encuentran en la bibliografia relacionados con el aprendizaje de las ciencias (química) utilizando nuevas tecnologias de la información y la comunicación; destacamos los siguientes:

Raviolo (2002) discute las posibilidades del Excel, o de otra hoja de cálculo, en la enseñanza de las ciencias y comparte los resultados de una experiencia didáctica llevada a cabo en cursos de química general. Este tipo de actividades, pueden servir como guía para los docentes, de esta o cualquier asignatura, que intenten implementar actividades de enseñanza con la computadora.

Bekerman et al., (2002) plantean la elaboración y aplicación de presentaciones animadas en computador para la enseñanza y técnica de recristalización, del concepto de azeótropos que corresponde a la técnica de destilación y de extracción continua. Instrumentos diseñados como tutoriales en el marco de la enseñanza técnica superior o universitaria básica. Este proceso se evalúa a través de encuestas a estudiantes.

Otra investigación relacionada con el diseño y aplicación de NTIC al modelo de enseñanza y aprendizaje por investigación, lo constituye el trabajo realizado por Ma Belén Garrido (1999) de la Universidad de Valencia, al diseñar un Programa de Actividades Hipermedia para el Aprendizaje de la Geometría y Polaridad de las Moléculas. El PGA consta de actividades, videos, moléculas tridimensionales, hipertexto, todo para el logro de aprendizaje significativo.

En el proyecto Red Internacional Virtual (RIVED, 2001-2002), adelantado por el Ministerio de Educación Nacional se hicieron reflexiones sobre la incorporación de NTIC en química, física y matemáticas, desde una perspectiva de resolución de problemas. De dicho proyecto se elaboraron 11 módulos multimedia, 4 de química, 3 de física y 4 de matemáticas. En el área de química se abordaron sustancias, mezclas y punto de ebullición y densidad, desde el modelo de enseñanza y aprendizaje por investigación. El proyecto tenía como propósito formar una red de profesores de diferen- 
tes países que trabajaran colaborativamente, una red de escuelas piloto y una red de producción de materiales. Quedó en su fase inicial.

\section{MARCOTEÓRICO}

Fundamentos de la resolución de situaciones problemas. Al tratar de explicar los fundamentos de la resolución de problemas desde la epistemología y la psicología como ejes centrales en el proceso de conocimiento y como proceso en sí mismo, el análisis epistemológico que se plantea incluye las conclusiones a las que han llegado filósofos de las ciencias como Popper, Kuhn y Toulmin. Las consideraciones desde la psicología que explican el proceso de resolución de situaciones problemas que se incluyen aquí son: la teoría asociacionista, la teoría de la Gestalt, la teoría del procesamiento de la información y la teoría del aprendizaje significativo que para efectos de nuestra investigación se hizo mayor énfasis.

Resolución de problemas y la enseñanza de las ciencias. Al establecer la relación entre los procesos de resolución de problemas y la enseñanza de las ciencias, encontramos dos perspectivas. La primera que concibe a la ciencia como el instrumento para desarrollar la capacidad de resolver problemas en los individuos. La resolución de problemas se considera como un fin y no como un medio para el aprendizaje, y la segunda que concibe el proceso de resolución de problemas como una herramienta útil para que los individuos aprendan ciencias, es decir, adquirir información utilizable a modo de instrumental conceptual que facilite la posterior resolución de problemas, información que pudiera aportar indicaciones adicionales sobre el significado (Bransford, Stein, 1993). Consideran además que "limitar el objetivo de la resolución de problemas al aprendizaje de heuristicas generales y especiales y a la aplicación de conocimientos previamente adquiridos supone desvincularla del proceso de construcción de los conceptos y teorias" (Gallastegui, 1989), y que el mejoramiento de las habilidades para resolver problemas en los estudiantes, mejorará el proceso de enseñanza y en particular de la educación en ciencias. Además, esta perspectiva que argumenta en favor de la utilización de los procesos de resolución de problemas para mejorar el aprendizaje de las ciencias tiene en cuenta que el objeto de la enseñanza debe involucrar otros elementos diferentes a la simple aprehensión de conocimientos científicos, como "el desarrollo de aptitudes, capacidades e intereses, de la autonomía, de la responsabilidad y del sentido crítico" (Sigüenza, Sáenz, 1990), que formen en el individuo "un modo de pensar que le permita resolver problemas por sí mismo" (Elstgreest, 1978) y que estos elementos pueden ser desarrollados en los individuos a través de los procesos de resolución de problemas.

Los estudiantes y la resolución de problemas. Cuando se pregunta a los estudiantes lo que sucede cuando ellos se enfrentan a la resolución de problemas su respuesta más común es "comprendo la teoría, pero ante un problema no tengo idea; entiendo los problemas, pero en cuanto se cambia algo del enunciado, no sé por dónde cogerlo" (Palacios, López Rupérez, 2000). Este estado de desconcierto puede estar causado por los procedimientos inadecuados que usualmente sigue el estudiante para resolver los problemas. Las características de estos procedimientos son las siguientes:

La mayoría de los estudiantes, no utilizan representaciones válidas del problema ni de las posibles vías para su resolución antes de iniciar esta resolución. Es así como "la mayoría de los estudiantes no elaboran gráficos o diagramas para ayudarse en la comprensión del problema y la mayoría de ellos tampoco piensa acerca de las diferentes formas y métodos que pueden existir para resolver un problema (Fortunato, Álvarez, 2000).

Cuando los estudiantes resuelven problemas en la clase de ciencias lo hacen de una forma mecánica sin tener en cuenta el porqué utilizan uno u otro proceso en la resolución, por ello, los estudiantes rara vez pueden ser capaces de dar una justificación coherente para utilizar este procedimiento en la resolución del problema.

- La mayoría de los estudiantes necesitan los conceptos, las leyes y las fórmulas para la resolución de problemas y le "dan una importancia predominante a encontrar una fórmula o un algoritmo" (Nakhleand, 1999); debido a esto "muchos de ellos no utilizan los conceptos para resolver los problemas conceptualmente y resuelven los problemas como ecuaciones algebraicas" Nakhleand (1999) entre otros.

Redes y estructuras objeto de enseñanza sistemas conceptuales. Ausubel (1978), el conocimiento previo del estudiante es el factor aislado 
más importante que influye en el aprendizaje, de allí que el papel crucial de la estructura cognoscitiva (complejo de conceptos jerárquicamente organizados) preexistente en el aprendizaje subsiguiente lleven a "descubrir lo que el alumno ya sabe y enseñarlo de acuerdo con eso".

Redes conceptuales. Galagotski (1994), propone un nuevo esquema que le llamo red conceptual, el cual presenta las siguientes características:

- El concepto de oración nuclear como recurso comunicacional entre los nodos.

- Flexibilizar la confección de la red de conceptos de forma tal que su disposición gráfica resulte independiente de la estructura jerárquica del tema.

Por tanto, construir una red conceptual es reconstruir explícitamente el tema desde los conceptos subyacentes hacia los nuevos conceptos.

Precisiones para la confección de una red conceptual. El sustento teórico imprime grandes exigencias para la confección de las redes conceptuales. Los requisitos establecidos para la confección de una red conceptual son los siguientes:

a) Los nodos de la red serán ocupados por signos lingüísticos que representen conceptos esenciales del tema en cuestión.

b) Conceptos muy abarcativos tales como "crisis de", "modelo de", etc, deberán corresponder al título de la red y no ser incorporados como nodos.

c) La totalidad de las uniones que relacionan conceptos deberán exhibir leyendas que incluyan un verbo preciso, de tal forma de generar una oración nuclear entre nodos.

d) Verbos tales como "afectan", "modifican", "está conectado con", "está relacionado con", etc., no son considerados precisos.

e) Las oraciones nucleares se leerán con un sentido señalado por una flecha.

f) No podrá construirse una oración cuyo significado se extienda entre más de dos nodos.

g) La lectura de la red podrá comenzar por cualquier nodo.

h) Se considerará artificial, la ordenación jerárquica de conceptos en relación con una disposición grafica vertical. El nuevo diseño gráfico sólo requiere claridad para la lectura.

i) Se consideran como conceptos fundamentales a aquellos a los que llegan y de los que parten la mayor cantidad de relaciones (flechas). Es- tos conceptos muy relacionados pueden ser, o no, los conceptos de jerarquía más abarcativa.

j) No se aceptará la repetición de conceptos (nodos).

k) No se incluirán en las leyendas sobre las flechas conceptos que pertenezcan a la esencia del tema en cuestión y que no hubieran sido desglosados previamente como nodos.

1) No se aceptarán ecuaciones matemáticas como nodos, excepto unidos a algún concepto mediante la leyenda "se simboliza mediante".

Algunos aspectos comparativos entre un mapa conceptual y una red conceptual aparecen en la Tabla 1.

Red conceptual ácido-base. La Gráfica 1 muestra la red conceptual sobre conceptos ácidobase, a que se pretendía acercar a los estudiantes objeto de esta investigación.

Esta red conceptual se construyó a partir del desglosamiento del concepto de ácido en ácido, acidez y solución ácida y su correspondencia con el concepto básico en básico, basicidad o alcalinidad y solución básica, desglosamiento que se realizó uniendo oraciones nucleares pares de conceptos, utilizando verbos que permitieran transformar una oración de estructura semántica superficial en una oración nuclear. La inclusión de ejemplos (nodos marcados en rojo, verde y violeta en la red) permite a los alumnos captar la idea sobre el carácter removible de ejemplo, pero perdurable del concepto. Para propósito de esta investigación, se abordó el aprendizaje significativo de los conceptos asociados con esta red conceptual ácido-base, abordándola lo más general posible.

\section{¿QUE SON LAS NTIC Y QUE CAMBIOS PRODU- CEN?}

Gilbert et al. (1992) han catalogado las NTIC como "el conjunto de herramientas soportes y canales para el tratamiento y acceso a la información". Otros señalan que se refieren a los últimos desarrollos tecnológicos y sus aplicaciones (Bartolomé, 1989). Desde otros puntos de vista las NTIC son catalogadas como una serie de aplicaciones de descubrimientos científicos cuyo núcleo central consiste en una capacidad cada vez mayor de tratamiento de la información". Existen algunas otras definiciones para el término. En esta investigación se entienden como aquellas herramientas, que nos permiten no sólo 
Tabla 1

Cuadro comparativo entre una red conceptual y un mapa conceptual

\begin{tabular}{|c|c|c|}
\hline Aspectos & Mapas conceptuales & Redes conceptual \\
\hline Nodos & $\begin{array}{l}\text { Se completan con sustantivos, verbos o } \\
\text { adjetivos. Se admiten expresiones mate- } \\
\text { máticas. }\end{array}$ & $\begin{array}{l}\text { Se complementan con sustantivos }+ \text { adjeti- } \\
\text { vos, que sean conceptos relevantes del } \\
\text { tema. La repetición de nodos esta prohi- } \\
\text { bida. No se aceptan fórmulas matemáticas, } \\
\text { excepto unidas por la leyenda «se simboliza } \\
\text { mediante». }\end{array}$ \\
\hline $\begin{array}{l}\text { Leyendas que unen } \\
\text { los nodos }\end{array}$ & $\begin{array}{l}\text { Se utilizan palabras para formar proposicio- } \\
\text { nes entre nodos. La extensión de una } \\
\text { oración puede abarcar más de dos nodos } \\
\text { consecutivos. }\end{array}$ & $\begin{array}{l}\text { Se utilizan palabras que completen una } \\
\text { oración nuclear entre nodos consecutivos. } \\
\text { Las oraciones nucleares se leerán siguiendo } \\
\text { el recorrido de una flecha.Las leyendas no } \\
\text { podrán contener conceptos relevantes que } \\
\text { no hubieran sido desglosados previamente } \\
\text { como nodos. }\end{array}$ \\
\hline Jerarquía gráfica & $\begin{array}{l}\text { Es absolutamente necesaria. Esta jerarquía } \\
\text { debe reflejar la jerarquía conceptual } \\
\text { específica del tema. }\end{array}$ & $\begin{array}{l}\text { No es necesaria. Los conceptos más impor- } \\
\text { tantes son los más relacionados }\end{array}$ \\
\hline
\end{tabular}

no van a manejar esta tecnología bien porque no quierano simplemente porqueles intimida" (Esebbag etal., 1996).

El planteamiento anterior nos lleva a pensar acerca del desfase entre la escuela y las NTIC. Mientras los docentes nos encontramos en las primeras experiencias de introducción e incorporación en los currículos de estos medios, los estudiantes viven fuera del contexto escolar, tanto de educación superior como de educación media, una familiarización con la tecnología bastante rápida. Productos más llamativos y mejor producidos, llevan a que los estudiantes se distraigan constantemente de sus labores.

Lo anterior sugiere una urgente necesidad de preparar ciudadanos competitivos para el nuevo milenio, que estén familiarizados con los lenguajes de comunicación e información, que cada vez avanzan rápidamente, para no tener sociedades analfabetas en un futuro próximo. Por parte de los profesores de todas las áreas, se genera el reto de mejorar la calidad de la enseñanza que se imparte, lo cual involucra la introducción y la utilización de nuevas tecnologías en el aula de clase. Esto hace que los profesores de secundaria y educación superior se capaciten en el uso, y porque no, en la producción de dichas herramientas.

Según Zangara (1998) hay tres revoluciones en el área de conocimiento que afectan a la educación superior

- Revolución científico tecnológica, unión entre ciencia y tecnologia, su dependencia.

- La ruptura de fronteras nacionales para la educación, educación a distancia, convenios interinstitucionales, avances en comunicaciones y transportación.

- Globalización de la economía, lo que implica nuevos escenarios de interacción y en el caso de la educación nuevos entornos de enseñanza y aprendizaje reales y virtuales.

En este sentido, se crea la necesidad urgente que la educación superior se encuentre a la vanguardia de una sociedad del conocimiento y acorde con los procesos comunicativos y económicos que plantea la economía mundial para ser competitivos.

\section{METODOLOGÍA}

Este trabajo se basó fundamentalmente en una metodología cualitativa de orientación interpretativa, que buscaba descubrir y explicar la realidad de las representaciones mentales de los estudiantes mediante la caracterización de las ideas alternativas y su integración en el proceso de enseñanza aprendizaje, dentro de un 


\section{Bioetnia Volumen 5 No 1 (enero-junio), 2008}

contexto de diseño, aplicación y evaluación de una página web en torno a los conceptos ácidobase, con el uso de situaciones problema como instrumento que potencie el aprendizaje significativo de los estudiantes en el área de química.

Para el diseño de la investigación se partió de:

- Definición de la muestra. Nivel educativo de los estudiantes a quienes van dirigidas las preguntas, la forma de aplicarlas y el modo de recoger la información.

- Instrumentación. Diseño y aplicación de los instrumentos para recoger la información.

- Identificación de algunas ideas alternativas, así como la caracterización de algunas de las herramientas que utilizan los estudiantes para resolver situaciones problemas sobre los conceptos ácido-base, mediante la aplicación de un cuestionario para detectar las ideas alternativas que presentaban los estudiantes objeto de este estudio en relación con los conceptos ácido-base y los procedimientos que utilizan para la resolución de una situación problema, llamado cuestionario 1, y un cuestionario de elaboración de redes conceptuales en torno a los conceptos ácido-base, denominado red conceptual 1.

- Diseño y elaboración de la página web de conceptos ácido-base, realizando la debida revisión bibliográfica requerida para poder satisfacer las necesidades con bases teóricas pedagógicas.

- Puesta en funcionamiento del sitio web para validarlo con expertos en el área tecnológica, expertos de contenido y usuarios.

- Aplicación del cuestionario 2, que en esencia es el mismo cuestionario 1, así como también se aplicó la reelaboración de la red conceptual 1 en torno a los conceptos ácido-base que hemos denominado red conceptual 2. Con la aplicación de estos instrumentos se conoció la evolución de las ideas alternativas en torno a los conceptos ácido-base, y la evolución de las habilidades para resolver problemas, y esto en esencia permitió medir el grado de aprendizaje significativo logrado por los estudiantes objeto de este estudio, sobre los conceptos ácidobase.

\section{DESARROLLODE LAINVESTIGACIÓN}

Esta estrategia se aplicó a los estudiantes de primer semestre del programa de Biología con
Énfasis en Recursos Naturales de la Universidad Tecnológica del Chocó "Diego Luis Córdoba", y se fundamentó en la determinación de algunas de las ideas alternativas, la resolución de situaciones problemas que utilizan los estudiantes, así como el diseño, aplicación y evaluación de una página web de conceptos ácido-base, con el objeto de lograr un aprendizaje más significativo en torno a los conceptos ácido-base por parte de los estudiantes objeto de la investigación. La investigación incluyó las siguientes fases:

Fase inicial. En la cual se aplicó a los estudiantes una prueba de ideas previas, esto con el objeto de conocer algunas de las ideas alternativas que presentan los estudiantes acerca de los conceptos ácido-base, el estado inicial de los estudiantes en términos de resolución de situaciones problemas y nivel de asimilación conceptual (construcción de una red conceptual).

Fase de acomodación al cambio didáctico. En esta fase, se explicó a los estudiantes la naturaleza del modelo didáctico y se diseñó y evalúo (juicio de expertos) la página web de conceptos ácido-base a utilizar, al igual que la estructura conceptual y problémica a desarrollar, se organizaron los equipos de estudiantes y se definieron las formas de trabajo.

Fase de ejecución. En esta fase se procedió a llevar a cabo los procesos de resolución de las situaciones problemas (problemas abiertos) propuestas por el profesor y los estudiantes, así como los procedimientos diseñados, respuestas obtenidas y se hicieron las clarificaciones conceptuales necesarias (implementación de la página web de conceptos ácido-base).

Fase de evaluación. Finalmente en esta fase, se aplicaron las mismas pruebas iniciales para determinar el cambio producido por la aplicación del modelo didáctico en las variables ideas alternativas, asimilación conceptual y de resolución de situaciones problemas.

Análisis de resultados de evaluación de la página web. $\mathrm{E} 1$ análisis de los resultados de la evaluación de la página web de conceptos ácidobase, cuya dirección electrónica es: http:/ /www. utch.edu.co/conceptosacido-base, se llevo a cabo mediante la organización de los resultados obtenidos mediante la realización del juicio de expertos. Este juicio de expertos que como ya lo hemos mencionado consistió en la aplicación de instrumento llamado: "Ficha de catalogación y evaluación con propuestas didáctica para espacios web 
Modelo de enseñanza y aprendizaje. CA Rentería

Tabla 2

Resultados totales arrojados por el juicio de expertos

\begin{tabular}{|c|c|c|c|c|c|c|}
\hline \multirow[b]{2}{*}{ Experto } & \multicolumn{3}{|c|}{ Aspectos } & \multirow[b]{2}{*}{$\begin{array}{l}\text { Contenidos } \\
\text { (36 pts) }\end{array}$} & \multirow[b]{2}{*}{$\begin{array}{c}\text { Valoración global } \\
\text { (12 pts) }\end{array}$} & \multirow[b]{2}{*}{$\begin{array}{l}\text { Total } \\
\text { (124 pts) }\end{array}$} \\
\hline & $\begin{array}{l}\text { funcionales } \\
\text { (24 pts) }\end{array}$ & $\begin{array}{c}\text { técnicos y estéticos } \\
\text { ( } 36 \mathrm{pts})\end{array}$ & $\begin{array}{l}\text { psicológicos } \\
\text { (16 pts) }\end{array}$ & & & \\
\hline 1 Experto & 19 & 26 & 14 & 30 & 9 & 98 \\
\hline 2 Experto & $\begin{array}{c}79 \% \\
21 \\
88 \%\end{array}$ & $\begin{array}{c}72 \% \\
27 \\
75 \%\end{array}$ & $\begin{array}{c}88 \% \\
15 \\
94 \%\end{array}$ & $\begin{array}{l}83 \% \\
34 \\
94 \%\end{array}$ & $\begin{array}{c}75 \% \\
10 \\
83 \%\end{array}$ & $\begin{array}{l}79 \% \\
107 \\
86 \%\end{array}$ \\
\hline 3 Experto & $\begin{array}{c}20 \\
83 \%\end{array}$ & $\begin{array}{c}30 \\
83 \%\end{array}$ & $\begin{array}{c}14 \\
88 \%\end{array}$ & $\begin{array}{c}32 \\
89 \%\end{array}$ & $\begin{array}{c}12 \\
100 \%\end{array}$ & $\begin{array}{l}108 \\
87 \%\end{array}$ \\
\hline 4 Experto & $\begin{array}{c}18 \\
75 \%\end{array}$ & $\begin{array}{c}32 \\
89 \%\end{array}$ & $\begin{array}{c}15 \\
94 \%\end{array}$ & $\begin{array}{c}34 \\
94 \%\end{array}$ & $\begin{array}{c}10 \\
83 \%\end{array}$ & $\begin{array}{l}109 \\
88 \%\end{array}$ \\
\hline 5 Usuario & $\begin{array}{c}22 \\
92 \%\end{array}$ & $\begin{array}{c}34 \\
94 \%\end{array}$ & $\begin{array}{c}14 \\
88 \%\end{array}$ & $\begin{array}{c}30 \\
83 \%\end{array}$ & $\begin{array}{c}10 \\
83 \%\end{array}$ & $\begin{array}{l}110 \\
89 \%\end{array}$ \\
\hline 6 Usuario & $\begin{array}{c}20 \\
83 \%\end{array}$ & $\begin{array}{c}30 \\
83 \%\end{array}$ & $\begin{array}{c}14 \\
88 \%\end{array}$ & $\begin{array}{c}34 \\
94 \%\end{array}$ & $\begin{array}{c}12 \\
100 \%\end{array}$ & $\begin{array}{l}110 \\
89 \%\end{array}$ \\
\hline 7 Usuario & $\begin{array}{c}19 \\
79 \% \\
83 \%\end{array}$ & $\begin{array}{c}28 \\
78 \% \\
82 \%\end{array}$ & $\begin{array}{c}16 \\
100 \% \\
\mathbf{9 1 \%}\end{array}$ & $\begin{array}{c}32 \\
89 \% \\
\mathbf{9 0} \%\end{array}$ & $\begin{array}{c}10 \\
83 \% \\
\mathbf{8 7} \%\end{array}$ & $\begin{array}{c}105 \\
86 \% \\
\mathbf{8 6 \%}\end{array}$ \\
\hline
\end{tabular}

de interés educativo" de Pere Marqués-UAB (2001).

En la Tabla 2 se muestran los resultados generales arrojados por el juicio de expertos, que incluye los aspectos funcionales, aspectos técnicos y estéticos, aspectos psicológicos, contenidos que presenta y valoración global de la página web.

En la Tabla 2 se observan los resultados en promedio para cada uno de los aspectos que se evaluaron. El puntaje final que se dio a través del promedio de los juicios de expertos para el sitio "Conceptos ácido-base" fue 86\%, catalogado como altamente recomendable para su uso.

Con respecto a los aspectos funcionales el resultado fue $83 \%$. Al referirse a los aspectos técnicos y estéticos, la puntuación promedio fue $82 \%$. Al tomar en cuenta los aspectos psicológicos, el sitio electrónico obtuvo una puntación de $91 \%$.

Con respecto a los contenidos el sitio obtuvo una puntuación de 90\%. En la validación global el sitio obtuvo una puntuación promedio de $87 \%$.

Se puede observar cómo la mayoría de las puntuaciones son bastante altas, de igual forma se ha decidido analizar cada uno de los aspectos tomados en cuenta, así como las recomendaciones realizadas por los expertos. Con base en estos resultados se realizarán modificaciones aptas, para que el recurso electrónico realmente tenga un valor agregado con respecto a los otros sitios y satisfaga las necesidades e intereses de los usuarios.

\section{CONCLUSIONES}

Las concepciones alternativas, detectadas en los estudiantes universitarios del programa de Biología, con Énfasis en Recursos Naturales, de la Universidad Tecnológica del Chocó "Diego Luis Córdoba", mostraron que estos alumnos identifican valores bajos del $\mathrm{pH}$, con ácidos "fuertes" y éstos como dañinos para el organismo; neutro con inocuo e inerte; concepción del carácter ácido o básico de una sustancia como algo inherente exclusivamente a dicha sustancia, etc. Establecen pocas diferencias entre los términos neutralización, hidrólisis, disociación, en los contextos cotidiano y científico.

Los resultados de la aplicación de la estrategia didáctica, basada en el modelo de enseñanza problémica, apoyada en entornos virtuales, permitió adelantar algunas conclusionesque debieron ser matizadas, teniendo en cuenta dos aspectos. En primer lugar, estos resultados se obtuvieron bajo condiciones experimentales; es decir, en condiciones de aula y ambientes de trabajo óptimos, o sea, más adecuados que los ambientes de clase habituales. Y en segundo lugar, que el tiempo de aplicación de 40 horas fue bastante 


\section{Bioetnia Volumen 5 No 1 (enero-junio), 2008}

reducido, para que las conclusiones aquí expuestas gocen de total validez. Esta salvedad deja la tarea de replicar la aplicación de la estrategia durante un período mucho mayor.

Los resultados obtenidos, a partir de la labor de seguimiento, mostraron que se puede lograr una mayor asimilación conceptual (aprendizaje significativo), en términos de capacidad argumentativa, autonomía, persistencia en el trabajo y nivel de comprensión de conceptos y procedimientos, pero también que el desarrollo de cada uno de estos indicadores, es de carácter progresivo. Esto deja en claro que el aprendizaje es un proceso y que su naturaleza es progresiva. Lo anterior es importante en la medida que permite comprender varios fenómenos, que se presentan cuando el estudiante aprende a través de una estrategia basada en la solución de problemas, apoyada en NTIC.

Las habilidades desarrolladas para resolver los problemas, muestran que la estrategia didáctica está orientada hacia el mejoramiento de la comprensión del problema, más que hacia la consecución de su solución. Esto se refleja en:

a) El desarrollo de la habilidad para la elaboración de gráficas, que mejora los niveles de representación del problema,

b) En el desarrollo de las habilidades para establecer las motivaciones a fin de resolver el problema y comprender los enunciados, lo cual favorece el aumento de los niveles de significado sobre el problema; y finalmente,

c) La disminución de la tendencia a resolver los problemas de manera mecánica y operativa, disminución que indica el desarrollo de los niveles de conciencia cognitiva en los estudiantes.

A partir de estos resultados, es posible afirmar, que la resolución de situaciones problemas, cualitativas y abiertas, favorece la comprensión conceptual, es decir, la construcción de los conceptos científicos más que el aprendizaje de procedimientos operativos, de tipo algebraico o matemático, para operar sobre cantidades y datos referidos a los conceptos científicos.

Además, es importante recalcar que la utilización de un sistema de autodirección como parte de la estrategia didáctica, como lo es la página web de conceptos ácido-base, con programas guía de actividades, cumple con dos cometidos básicos: el primero, proporcionar saber metacognitivo al estudiante, proveyéndole de una guía para pensar, que a la vez lo capacita en las diferentes habilidades requeridas para resolver problemas y mejora su capacidad para aprender; el segundo, facilitar al estudiante llevar a cabo los procesos de regulación metacognitiva, proporcionándole elementos para el reconocimiento y la valoración autónoma de su trabajo académico, y elevando de esta manera el nivel de conciencia sobre sus propios procesos de aprendizaje.

La utilización de redes conceptuales, por parte de los alumnos, los llevó a encontrar los conceptos fundamentales y a establecer las relaciones más relevantes en cada tema, más allá de la simple repetición de ejemplos aprendidos.

Es importante destacar que cada uno de los objetivos específicos se logró, dando como resultado la consecución del objetivo general, que se planteó al iniciar la investigación. Se elaboró un sitio web con recursos, actividades y estrategias para estudiantes y docentes de últimos grados de secundaria y primeros semestres universitarios, en el área de química general en torno al tema conceptos ácido-base.

El sitio web "Conceptos ácido-base", es el resultado de un exhaustivo proceso de investigación, que permitirá a docentes tener acceso a recursos, actividades, experimentos, lecturas, modelos de planificación, contenidos del currículo de química general, en especial de los conceptos ácido-base, satisfaciendo así las necesidades e intereses particulares y aprovechando al mismo tiempo, los recursos tecnológicos de manera útil y eficaz para el desarrollo profesional en la práctica pedagógica.

Esta investigación, podrá dar cabida a nuevos espacios para el desarrollo de otros recursos relacionados con la tecnología educativa. A futuro, esta investigación puede ser retomada por estudiantes o profesionales, con el fin de reacomodarse a nuevas tendencias, necesidades e intereses.

Definitivamente, este sitio web servirá de apoyo en la labor pedagógica enriqueciendo el proceso de enseñanza-aprendizaje.

\section{LITERATURA CITADA}

Alarcón, A., Espitia, J., y Muñoz, G. 1988. Estudio de las incidencias de las ideas intuitivas en la aprehensión de los conceptos científicos de ácido y base. Alumnos de grado Décimo de Educación Media, Universidad Pedagógica Nacional, Facultad de Ciencia y Tecnologia, Departamento de Quimica, Santa Fe de Bogotá.

Arrieta Gallastegui, J. 1989. La resolución de problemas y la educación matemática: hacia una mayor interrelación entre 


\section{Modelo de enseñanza y aprendizaje. CA Rentería}

investigación y desarrollo cunicular. Enseñanza de las Ciencias. 7 (1): 63-71.

Ausubel, D., Hanesian, H., Novack, J.D. 1986. Psicología cognitiva un punto de vista cognoscitivo. México: Editorial Trillas.

Bransford, J.D. y Stein, B.S. 1993. Importancia de la heurística: solución ideal de problemas. New York: Editorial Labor S.A.

Bekerman, D. y Galagovsky, L. 2005. Tema soluciones: detección de dificultades en el procesamiento de información verbal y gráfica. IV Jornadas de Enseñanza Universitaria y preuniversitaria de la Química, UNAM, Mérida, México. Noviembre 14-17.

Elstgreest, J. 1978. La enseñanza de las ciencias mediante la resolución de problemas. Revista Perspectivas. 8 (1): 69-76.

Galagovsky, L. Kurgan, L.R. 1996. Redes conceptuales: Aprendizaje, comunicación y memoria. Bueno Aires: Editorial Lugar.

Hartley, J. R. 1988.Learning from computer based in learning in science. Studies in Science Education. 15: 55-76.

Izquierdo, M., Sanmartí, N., Espinet, M. y Garcia, P. 2000.
Application of a model shift of scientific knowledge: From the metaphor of the "book" to the metaphor of "discourse". Comunicación presentada en la 25 Conferencia Internacional de la ATEE. Barcelona.

Kunt, T. 1996. La estructura de las revoluciones científicas. Santafé de Bogotá: Editorial Fondo de Cultura Económica.

Lakatos, I. 1983. La metodología de los programas de investigación científica. Madrid: Alianza.

Lelouche, R. 1998. How education can benefit from computer: A critical review. Procedings of IV International Conference CALISCE '98. Donostia.

Popper, K. 1991. La lógica de la investigación científica. México: Editorial Rei. p. 262.

Raviolo, A. 2002. Hojas de cálculo en clases de ciencias. JSciEduc. 3 (2): $100-2$.

Sigüenza, A.F. y Sáenz, M.J. 1990. Análisis de la resolución de problemas como estrategia de enseñanza de la Biología. Enseñanza de las Ciencias. 8 (3): 223-30. 